\title{
Bewirtschaftung alpiner Speicherkraftwerke unter Berücksichtigung klimatischer und wirtschaftlicher Veränderungen
}

\author{
Die Wasserkraft ist mit großen Herausforderungen (Klimawandel und Strommarktliberalisie- \\ rung) konfrontiert. Ein neuartiger integrativer Ansatz erlaubt die Modellierung von komplexen \\ Wasserkraftanlagen zur Beurteilung der Auswirkungen auf deren Betrieb. Das Simulations- \\ programm Routing System beinhaltet nebst einem hydrologischen Modell für alpine Ein- \\ zugsgebiete auch ein Betriebsmodul für Speicherkraftwerke.
}

\section{Einleitung}

In Europa wird mehr als $40 \%$ des Stroms aus Wasserkraft im Alpenraum produziert [5]. Die alpinen Speicherkraftwerke sind in der Lage, die unregelmäßig anfal- lende Elektrizitätsproduktion aus erneuerbaren Quellen im europäischen Verbundnetz aufzuwerten. Um eine nachhaltige Bewirtschaftung zu gewährleisten, gilt es, sich der Umverteilung der Wasserressourcen durch den Klimawandel so-

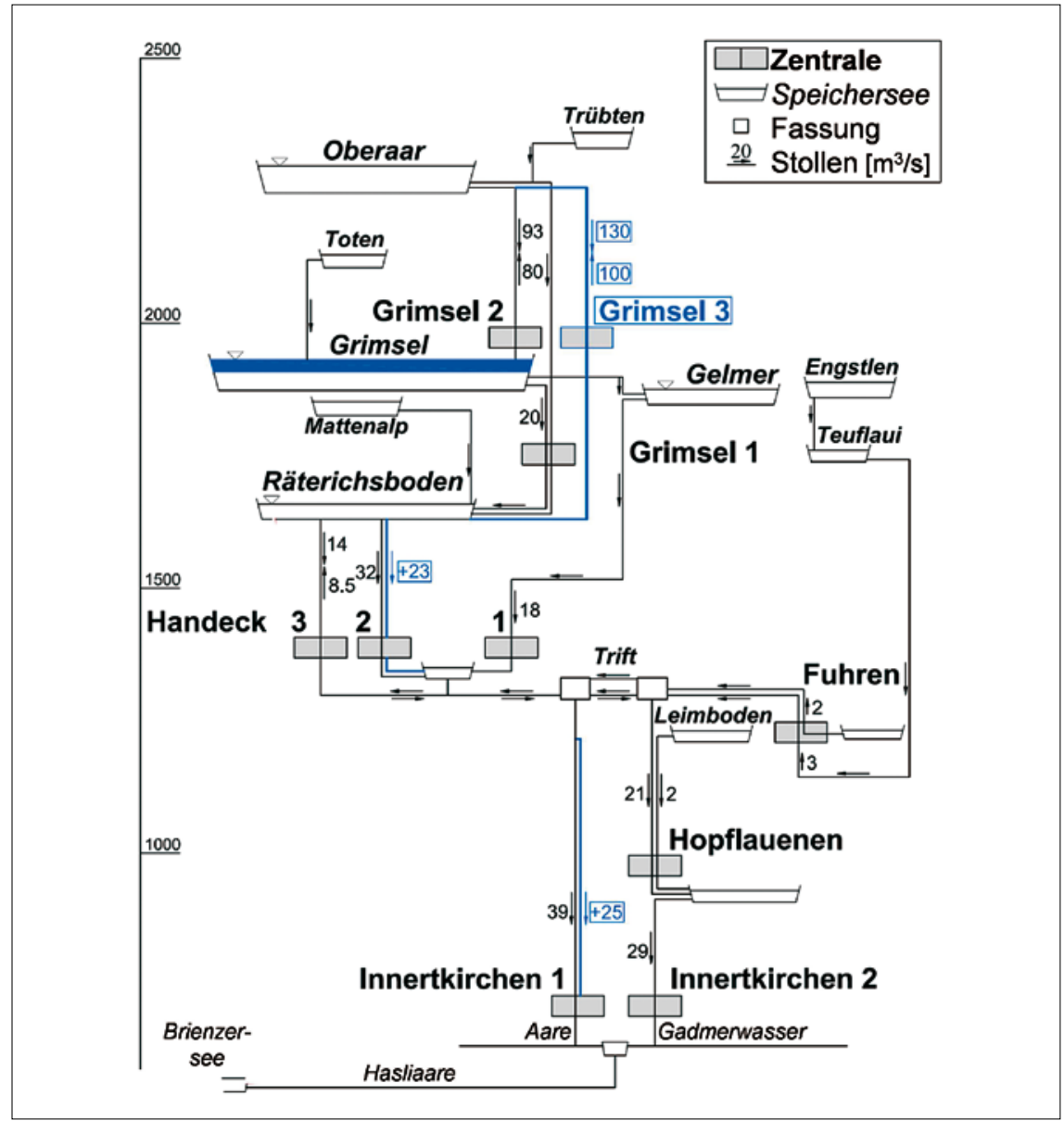

Bild 1: Heutiger Kraftwerkskomplex der Kraftwerke Oberhasli AG (KWO) mit Zentralen, Speichern, Wasserfassungen und Stollen, sowie den Ertüchtigungsmaßnahmen des Ausbauprojekts KWOplus (in blau). wie den veränderten rechtlichen, politischen und wirtschaftlichen Rahmenbedingungen zu stellen. Zur Evaluation der wechselnden Parameter wurde das integrative Simulationsprogramm Routing System entwickelt [2]. Es beinhaltet für die Modellierung von Speicherkraftwerken in alpinen Einzugsgebieten nebst einem hydrologischen Modell auch ein Betriebssimulationstool, welches Strompreisszenarien berücksichtigt.

\section{Fallstudie}

Als Fallstudie dient das Einzugsgebiet der Hasliaare in der Schweiz. Das Einzugsgebiet der Aare oberhalb Brienzwiler erstreckt sich über $554 \mathrm{~km}^{2} .2003$ waren $21 \%$ der Fläche vergletschert. Die Aare entspringt dem Unter- und Oberaargletscher auf rund $2000 \mathrm{~m}$ ü. M. und durchfließt die Speicherseen Oberaar, Grimsel sowie Räterichsboden. Rund 60 \% des Einzugsgebietes sind von einem Kraftwerkskomplex mit acht Speicherseen und neun Zentralen der Kraftwerke Oberhasli AG bewirtschaftet (Bild 1), darunter das Umwälzwerk Grimsel 2 zwischen dem Oberaar- und dem Grimselsee. Die Gesamtleistung aller Turbinen beträgt rund 1000 MW, jene der installierten Pumpen 400 MW. Seit den 30er Jahren nimmt der saisonale Wassertransfer vom Sommer in den Winter durch Kraftwerksausbauten stetig zu. Das Ausbauprojekt KWOplus, das bis Ende 2019 realisiert wird, soll die Flexibilität des Kraftwerks künftig erhöhen. Dabei soll die Speicherkapazität des Grimselsees von heute 100 auf 172 Mio. $\mathrm{m}^{3}$ erhöht, die Kraftwerke Handeck 2 und 
Innertkirchen 1 um 240 MW ertüchtigt und ein neues 600-MW-Pumpspeicherkraftwerk Grimsel 3 zwischen Oberaarund Räterichsbodensee zugebaut werden (Bild 1, in blau).

\section{Methode}

Die Simulation hydrologischer Prozesse in Routing System basiert auf einem konzeptionellen Modellierungsansatz und beinhaltet das speicherbasierte NiederschlagAbfluss-Transformationsmodell GSMSOCONT [4]. Räumliche und zeitliche Niederschlags- und Temperaturverteilungen bestimmen Schnee- resp. Gletscherbildung und -schmelze sowie den Grundwasser- und Oberflächenabfluss [1]. Das Einzugsgebiet wird in Untereinzugsgebiete und diese wiederum in vergletscherte und unvergletscherte Höhenbänder aufgeteilt. Für jedes Höhenband werden die meteorologischen Eingangsgrößen aus den Datenreihen der im Einflussradius gelegenen Messstationen errechnet. Die Berücksichtigung des höhenabhängigen Temperaturgradienten ermöglicht die Unterscheidung zwischen Fest- und Flüssigniederschlag.

\section{Modellierung}

Nebst den Retentionsprozessen in den Fließgewässern werden sämtliche Funktionen der hydraulischen Anlagen simuliert. Ein heuristischer Modellierungsansatz wurde zur Optimierung des Betriebs von Kraftwerkskomplexen implementiert, der einerseits den kurz- sowie langfristigen Zufluss und andererseits die Strom- preise berücksichtigt. Um den maximalen Ertrag aus dem Kraftwerksbetrieb zu bestimmen, muss ein nichtlineares Gleichungssystem gelöst werden, das die Betriebsstunden der Zentralen festlegt. Zur Lösung dieses Problems wurde ein deterministisch-heuristischer Ansatz gewählt.

Die Definition des Kraftwerkskomplexes in Routing System geschieht über eine grafische Benutzeroberfläche, wo sämtliche Komponenten angeordnet und bestimmt sowie deren Verbindungen charakterisiert werden. Für die Simulation werden die ausgewählten Zeitreihen, wie z. B. Strompreis oder Zufluss, aus einer Datenbank entnommen. Bei einfachen Einzugsgebieten kann der hydrologische Teil auch direkt mit dem Kraftwerk gekoppelt werden ohne Zugriff auf die Datenbank. Eine realitätsnahe Simulation beinhaltet sowohl kurz- als auch langfristige Aspekte. So müssen einerseits die saisonalen (Füllen des Stausees im Sommer bei tiefen Strompreisen für den Betrieb im Winter mit hohen Strompreisen) sowie die Gegebenheiten aus dem Tagesbetrieb für Strompreis und Zufluss berücksichtigt werden. Im vorliegenden Fall wurden jeweils fünf Betriebsjahre mit einem stündlichen Zeitschritt simuliert. Jeder Speicher wird mit dessen Füllkurve für das zu bewirtschaftende Volumen definiert. Die wichtigsten Parameter der Zentralen sind die Turbinen- sowie Pumpkapazitäten, die jeweils voll oder gar nicht ausgenutzt werden.

Der Tagesbetrieb muss stets auf dessen langfristigen Auswirkungen evaluiert werden. Darum wird für jeden Speichersee eine Zielfüllkurve bestimmt, welche auf statistischen Zufluss- und Preiskenn-

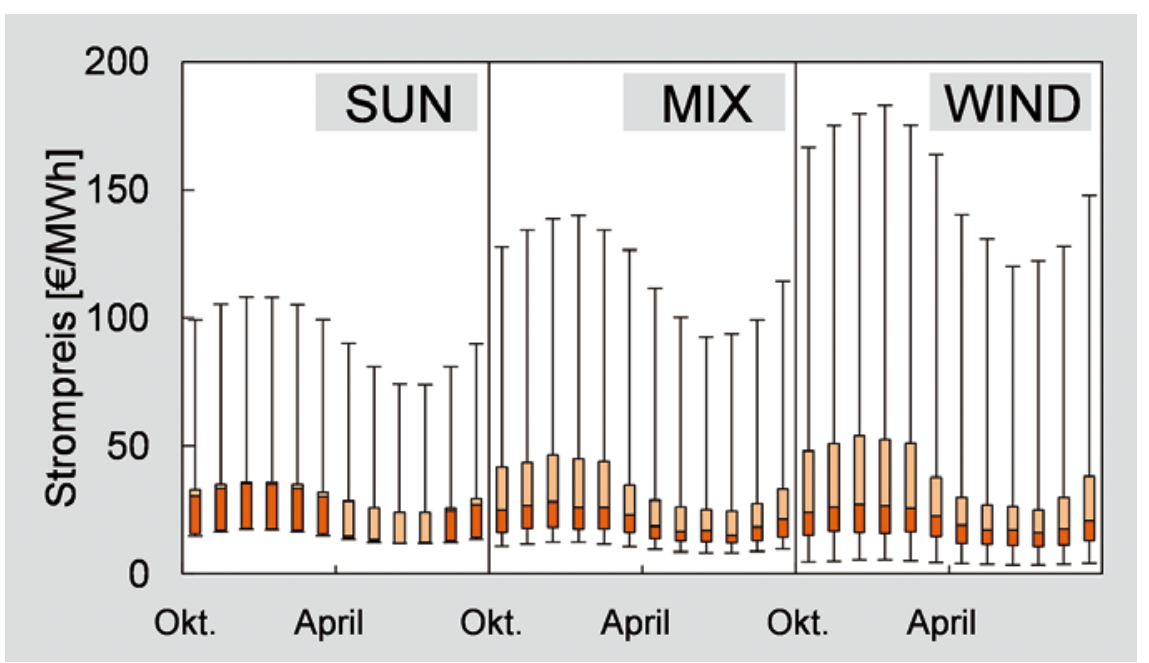

Bild 2: Statistische Auswertung (Extrema, 25-\%- und 75-\%-Perzentile) der Stundenstrompreise der Szenarien mit tiefer (SUN), mittlerer (MIX) und hoher (WIND) Volatilität.

werten beruht. Ein benutzerdefinierter Parameter gibt an, wie strikt diese vorgegebene Füllkurve eingehalten werden muss. Je nach Unsicherheit der Angaben kann dieser höher oder tiefer gesetzt werden. Für den Tagesbetrieb wird ein Zeithorizont bestimmt, für den die Strompreise sowie Zuflüsse bekannt sind. Bei jedem Zeitschritt werden diese Angaben aus der Datenbank für die Optimierung beigezogen. Ob für einen gegebenen Zeitpunkt eine Turbine (oder Pumpe) in Betrieb ist, wird wie folgt bestimmt:

1. Es wird abgeklärt, ob es im vorgegebenen Zeithorizont zu einem Überlaufen des oberhalb liegenden Speichers kommen könnte. Ist dies der Fall, so wird der Zeithorizont der Betriebsoptimierung verkürzt. Je nach Bedarf können bereits präventive Maßnahmen definiert werden.

2. Wenn das vorhandene Volumen im Speichersee gering ist, so wird nur jene Anzahl Produktionsstunden betrachtet, die noch eine Volllast der Turbine zulassen würde.

3. Sofern das Triebwasser in einen anderen Speicher eingeleitet wird, muss abgeklärt werden, ob genug Leervolumen vorhanden ist. Sollte dies nicht der Fall, so wird die Anzahl Produktionsstunden eingeschränkt.

4. Entsprechend der bestimmten Anzahl Betriebsstunden werden nun für den gewählten Zeithorizont jene Stunden mit dem höchsten Strompreis für den Betrieb vorgesehen. Der tiefste Preis der ausgesuchten Betriebsstunden gilt als Grenzpreis.

5. Ein Koeffizient wird bestimmt, der das Verhältnis zwischen aktuellem Füllstand und Zielfüllstand der Zielfüllkurve berücksichtigt.

6. Ist der Strompreis für den gegebenen Zeitschritt sowohl höher als der Grenzpreis als auch das Produkt aus Gestehungspreis und Füllstandskoeffizienten, so wird die Turbine mit Volllast betrieben. Andernfalls bleibt die Produktion für den Zeitschritt aus.

Der vom Benutzer zu bestimmende Gestehungspreis sowie der Parameter, wie strikt diese vorgegebene Füllkurve eingehalten werden muss, erlauben eine Kalibrierung des Modells für den heutigen Zustand.

\section{Szenarien}

Die simulierten Klimaszenarien basieren auf einer Referenzperiode (1980 bis 2009) und berücksichtigen künftige jährliche 
Tab. 1: Mittlere Jahresstromproduktion und -verbrauch sowie Betriebsertrag für das heutige Kraftwerk sowie das Ausbauvorhaben KWOplus für die drei Strompreisszenarien SUN, MIX und WIND und die drei Zeitabschnitte 2005 bis 2009, 2046 bis 2050 und 2095 bis 2099.

\begin{tabular}{|c|c|c|c|c|c|c|c|c|c|c|c|}
\hline \multirow{2}{*}{\multicolumn{2}{|c|}{$\begin{array}{l}\text { Zeitabschnitt } \\
\text { Strompreisszenario }\end{array}$}} & & \multicolumn{3}{|c|}{2005 bis 2009} & \multicolumn{3}{|c|}{2046 bis 2050} & \multicolumn{3}{|c|}{2095 bis 2099} \\
\hline & & & SUN & MIX & WIND & SUN & MIX & WIND & SUN & MIX & WIND \\
\hline \multirow{7}{*}{ 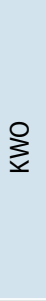 } & Produktion & [TWh/Jahr] & 2,2 & 2,5 & 2,6 & 2,0 & 2,3 & 2,4 & 1,8 & 2,1 & 1,8 \\
\hline & ... im Winter & [\%] & 46 & 44 & 44 & 57 & 54 & 52 & 59 & 54 & 59 \\
\hline & Verbrauch & [TWh/Jahr] & $-0,7$ & $-1,1$ & $-1,2$ & $-0,7$ & $-1,1$ & $-1,3$ & $-0,7$ & $-1,2$ & $-0,7$ \\
\hline & ... im Winter & [\%] & 71 & 58 & 54 & 70 & 57 & 52 & 66 & 53 & 66 \\
\hline & Gesamtproduktion & [TWh/Jahr] & 1,5 & 1,4 & 1,4 & 1,3 & 1,2 & 1,1 & 1,1 & 0,9 & 1,1 \\
\hline & Ertrag & [Mio. €/Jahr] & 108 & 118 & 127 & 105 & 112 & 123 & 101 & 109 & 116 \\
\hline & ... im Winter & [\%] & 66 & 66 & 64 & 74 & 72 & 70 & 74 & 71 & 70 \\
\hline \multirow{7}{*}{ 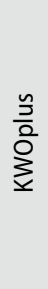 } & Produktion & [TWh/Jahr] & 3,1 & 3,7 & 3,9 & 3,1 & 3,6 & 3,7 & 3,0 & 3,7 & 3,8 \\
\hline & ... im Winter & [ \%] & 58 & 54 & 52 & 65 & 59 & 58 & 63 & 57 & 56 \\
\hline & Verbrauch & [TWh/Jahr] & $-2,0$ & $-2,8$ & $-3,1$ & $-2,2$ & $-2,9$ & $-3,1$ & $-2,4$ & $-3,3$ & $-3,5$ \\
\hline & ... im Winter & [\%] & 69 & 58 & 55 & 68 & 57 & 55 & 63 & 53 & 52 \\
\hline & Gesamtproduktion & [TWh/Jahr] & 1,1 & 0,9 & 0,8 & 0,9 & 0,7 & 0,6 & 0,6 & 0,4 & 0,3 \\
\hline & Ertrag & [Mio. €/Jahr] & 125 & 173 & 190 & 162 & 174 & 189 & 157 & 173 & 190 \\
\hline & ... im Winter & [\%] & 65 & 74 & 71 & 78 & 77 & 75 & 76 & 75 & 73 \\
\hline
\end{tabular}

und zwischenjährliche Niederschlagsund Temperaturänderungen bis 2099. Die Massenbilanzen der großen Gletscher sowie der daraus resultierende Tagesabfluss wurden in stündlichen Zeitschritten mit dem geeichten hydrologischen Modell für sämtliche Untereinzugsgebiete berechnet. Das hier diskutierte Klimaszenario ohne verringerten $\mathrm{CO}_{2}$-Ausstoss A1B errechnet für das alpine Einzugsgebiet bis 2099 einen Temperaturanstieg um $4{ }^{\circ} \mathrm{C}$ sowie einen höheren Niederschlag im Frühsommer und Spätherbst, jedoch einen Rückgang im August und September [3].

Der Betrieb des heutigen Kraftwerks und des Ausbauvorhabens KWOplus wurde für die generierten Zuflüsse sowie drei Preisszenarien identischer Monatsmittelwerten, jedoch unterschiedlicher Volatilität simuliert (Bild 2):

- Szenario SUN basiert auf einer Stromversorgung mit größtenteils photovoltaischen Anlagen, was zu relativ langen und vorhersehbaren Spitzen an Sommertagen mit dementsprechend tieferen Strompreisen führt.

- Szenario WIND berücksichtigt einen großen Zubau von Windkraftanlagen. Die saisonal sowie auch kurzfristig stark schwankenden Windverhältnisse führen zu einem sehr volatilen Marktpreis.

- Szenario MIX sieht verschiedene Produktionsquellen im europäischen Verbund vor und ist somit ein Kompromiss zwischen den oben erwähnten Szenarien.

\section{Ergebnisse}

\section{Modellkalibrierung}

Die Kraftwerke Oberhasli AG hatte zwischen 2005 und 2009 eine mittlere Bruttostromproduktion von $1,54 \mathrm{TWh}$. Von den eigentlich produzierten 2,24 TWh wurden 0,69 TWh für das Pumpen, hauptsächlich durch das Umwälzwerk Grimsel 2, verwendet. $36 \%$ der Stromproduktion und $50 \%$ der Pumpenergie fiel im Winter an. Die mit Szenario SUN simulierten Werte (Tabelle 1) kommen den statistischen Angaben am nächsten. Der Produktionsertrag liegt mit 88 Mio. $€$ pro Jahr (113 aus dem Turbinenbetrieb und 25 zum Pumpen) tiefer als die simulierten 108 Mio. €. Dieser Vergleich ist je- doch nicht relevant, da der heutige Betrieb nur teilweise vom Spotmarkt getrieben ist und die meisten Lieferungen vertraglich festgelegt sind. Trotz vereinfachten Rand- sowie Anfangsbedingungen erlauben die Simulationen mit Routing System plausible Ergebnisse für einen liberalisierten Strommarkt.

\section{Simulation}

Das Szenario ohne Verringerung der $\mathrm{CO}_{2}-$ Emissionen hat ein nahezu vollständiges Abschmelzen der Eismassen im 21. Jahrhundert zur Folge. Die ausfallende Gletscherschmelze im Sommer sowie die früher und intensiver einsetzende Schneeschmelze ändern das Abflussregime der Hasliaare von heute glazio-nival zu nival

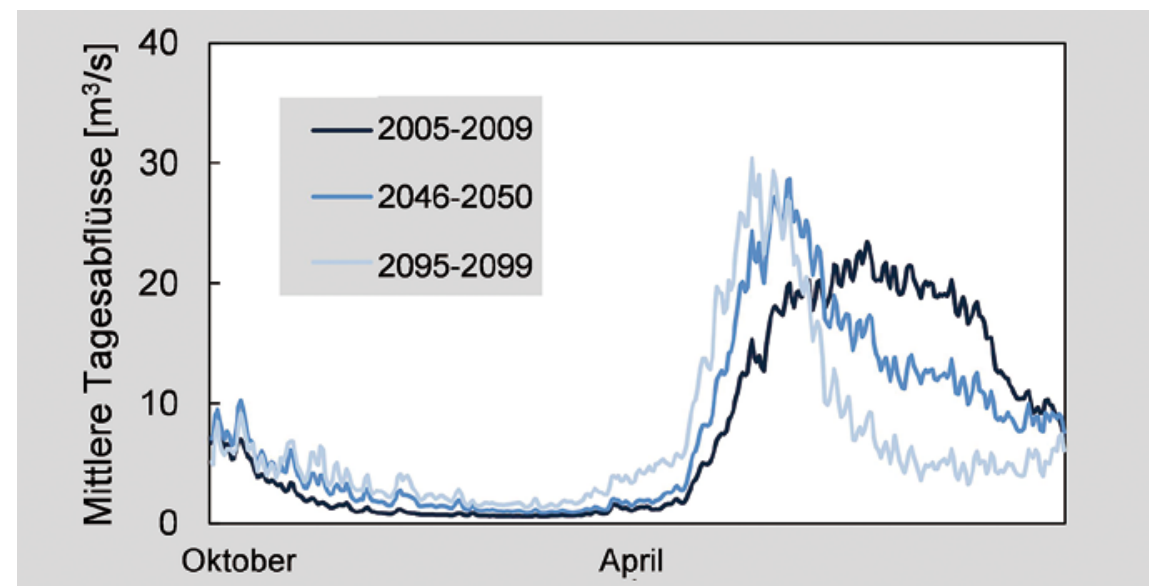

Bild 3: Mittlerer Tageszufluss des Betriebsgebietes der Kraftwerke Oberhasli AG für die drei Zeitabschnitte 2005 bis 2009, 2046 bis 2050 und 2095 bis 2099 basierend auf Klimaszenario ohne verringerten $\mathrm{CO}_{2}$-Ausstoss $\mathrm{A} 1 \mathrm{~B}$ [3]. 
(Bild 3). Die Zuflüsse zu den Speicherseen werden um $15 \%$ bis 2050 und um $25 \%$ bis Ende des Jahrhunderts geringer ausfallen als heute.

Konkret bedeutet dies für das heutige Kraftwerk ohne Umwälzwerke (Tabelle 1), dass die mittlere Jahresproduktion von 2,2 TWh zwischen 2005 und 2009 für das Szenario SUN auf 2,0 TWh bis Mitte dieses Jahrhunderts und auf 1,8 TWh bis zu dessen Ende zurückgehen wird. Dies entspricht Produktionsausfällen von rund $10 \%$ und $20 \%$. Die vergleichsweise großen Volumina der Speicherseen garantiert jedoch auch künftig eine hohe Winterproduktion von ca. 1,0 TWh für das heutige Kraftwerk und 1,8 TWh für KWOplus. Der Zubau von Turbinen und Pumpen erhöht sowohl Stromproduktion als auch den Pumpverbrauch. In Kombination mit einem größeren Speichervolumen des Grimselsees kann die Winterproduktion sogar leicht erhöht werden. Ohne das Ausbauprojekt würden der Turbinen- und der Pumpbetrieb aufgrund des frühen Füllens der Speicherseen während der Sommermonate eingeschränkt.
Dank der Betriebsflexibilität mit den bestehenden Pumpspeicheranlagen reduziert sich der Produktionsertrag trotz Zuflussrückgang nur um $4 \%$ bis Mitte des Jahrhunderts bzw. 8 \% bis zu dessen Ende. Der Zubau von Turbinen- sowie Pumpspeicherkapazitäten kann den Ertragsausfall weitgehend kompensieren und gar Mehreinnahmen generieren, sofern gleichzeitig die Volatilität der Strompreise zunimmt.

\section{Diskussion}

Ein heuristischer Modellierungsansatz wurde in Routing System zur Optimierung des Betriebs von Kraftwerkskomplexen verwendet. Im Rahmen der Fallstudie der Kraftwerke Oberhasli AG - und trotz der Komplexität des Systems - konnte der Einfluss der Klimaänderung, der Veränderungen im Strommarkt sowie von Ausbauvorhaben untersucht werden.

Routing System wurde auch zur Beurteilung der Hochwasserretentionskapazität von Speicheranlagen [1] sowie der Kosten-

Martin Bieri, Anton J. Schleiss and Frédéric Jordan

\section{Operation of Alpine Storage Hydropower Schemes under Climatic and Economic Changes}

Hydropower is the world's most important renewable energy source. High-head storage hydropower plants (HPP) contribute significantly to peak electricity production and grid regulation. Future plant management is faced with several challenges concerning modified availability of water resources due to climate change as well as electricity market issues. A novel integrative approach to model complex HPPs and assess the impact on their operation was developed. The simulation tool Routing System contains besides a precipitation-runoff model for long-term simulations of glacierized Alpine catchment areas an HPP operation tool.

Мартин Биери, Антон Й. Шлейсс и Фредерик Йордан

\section{Хозяйственное использование альпийских аккумулирующих гидроэлектростанций с учетом климатических и экономических изменений}

Гидроэнергетика испытывает сегодня серьезные проблемы (климатические изменения и либерализация энергетического рынка). Новый интегративный подход позволяет осуществлять моделирование целых ГЭС для оценки воздействия этих аспектов на их эксплуатацию. Программа моделирования «Routing System» включает в себя, наряду с гидрологической моделью альпийских водосборных бассейнов, модуль эксплуатации гидроаккумулирующих электростанций.

Nutzen-Beurteilung von baulichen wie auch betrieblichen Schwallsanierungsmaßnahmen [2] erfolgreich eingesetzt. Der entwickelte Ansatz ist ein praxisorientiertes Werkzeug um den künftigen Herausforderungen im Bereich Wasserkraft proaktiv und effizient zu begegnen.

\section{Danksagung}

Das Forschungsprojekt (9676.1 PFIW-IW) wurde von der Kommission für Technologie und Innovation (KTI) und der Kraftwerke Oberhasli AG (KWO) sowie weiteren privaten und öffentlichen Partnern finanziert.

\section{Autoren}

\section{Dr. Martin Bieri}

Pöyry Energy AG

Hardturmstrasse 161, CH-8037 Zürich

martin.bieri@poyry.com

\section{Prof. Dr. Anton J. Schleiss}

Laboratoire de Constructions Hydrauliques (LCH) Ecole Polytechnique Fédérale de Lausanne (EPFL) Station 18

CH-1015 Lausanne

anton.schleiss@epfl.ch

\section{Dr. Frédéric Jordan}

e-dric.ch ingénieurs conseils Sàrl

Chemin du Rionzi 54

CH-1052 Le Mont-sur-Lausanne

fred.jordan@e-dric.ch

\section{Literatur}

[1] Bieri, M.; Schleiss A. J.: Analysis of flood-reduction capacity of hydropower schemes in an Alpine catchment area by semi-distributed conceptual modelling. In: Journal of Flood Risk Management (2012), Online-Publikation (http://onlinelibrary.wiley.com/doi/10.1111/ j.1753-318X.2012.01171.x/abstract; Abruf 27.01.2013).

[2] Bieri, M.: Operation of complex hydropower schemes and its impact on the flow regime in the downstream river system under changing scenarios. In: Mitteilung (Communication) des Laboratoire de constructions hydrauliques (2012), LCH N 52 (Ich.epfl.ch).

[3] Bosshard, T.; Kotlarski S.; Ewen, T.; Schär, C: Spectral representation of the annual cycle in the climate change signal. In: Hydrology Earth System Sciences Discussions (2011), Heft 8, S. 1161-1192.

[4] Schaefli, B.; Hingray, B.; Niggly, M.; Musy, A.: A conceptual glacio-hydrological model for high mountainous catchments. In: Hydrology and Earth System Sciences (2005), Heft 9 (1), S. 95-109.

[5] Schleiss, A.: L'hydraulique suisse: Un grand potentiel de croissance par l'augmentation de la puissance. In : Bulletin SEV/VSE (2007), Heft 07 (2), S. 24-29. 